\title{
Diffusion of hydrogen in transition metals*
}

\author{
H. R. Schober \\ Institut für Festkörperforschung, Forschungszentrum Jülich, D-5170 Jülich (F.R.G.)
}

\author{
A. M. Stoneham \\ AEA Industrial Technology, Harwell, Oxfordshire OX11 ORA (U.K.)
}

\begin{abstract}
The mobility of interstitial atoms is closely correlated with their vibrational behaviour. Not only can the classical (hopping) activation energy be evaluated with good accuracy from the vibrational spectrum, but also low temperature tunnelling rates are strongly related to vibrations. Because of their small mass interstitial hydrogen atoms constitute an ideal probe to study this correlation over a large temperature range $(0 \mathrm{~K}<T<300 \mathrm{~K})$. Hydrogen atoms vibrate mainly with localized (optical) modes but also with strongly distorted lattice vibrations (short-wavelength acoustic modes). These vibrations lead to a "dressing" of the tunnelling element. More interesting, they determine the temperature dependence of the diffusion constant (at low temperatures together with electronic terms). Using a realistic description of the vibrations as input we calculate the diffusion constants quantitatively. We present results for hydrogen in niobium and in lutetium.
\end{abstract}

\section{Introduction}

The diffusion of hydrogen in metals has been studied extensively in the past both theoretically and experimentally [1] and is today still the subject of numerous investigations. Apart from the technological importance this is due to the small mass of the hydrogen which makes it an ideal probe to study quantum effects and the transition to classical mechanics. A particular advantage is the large mass ratio between the isotopes hydrogen, deuterium and tritium and $\mu^{+}$. Depending on temperature and concentration one finds a number of different phases of the hydrogen metal system. In this paper we shall restrict ourselves to the limit of low hydrogen concentration ( $\alpha$ phase) where the hydrogen atoms occupy random interstitial sites. Translational symmetry requires that the potential seen by a hydrogen atom has the periodicity of the lattice of interstitial sites. The potential energy can be thought of as consisting of two parts: the potential energy of the hydrogen in the undistorted, ideal metal lattice and an energy gain due to the relaxation of host atoms surrounding the hydrogen. This lattice relaxation around a given site destroys the periodicity of the lattice; the hydrogen cannot move to a neighbouring site conserving energy without distorting the lattice.

*Invited paper presented at the International Symposium on Metal-Hydrogen Systems, Banff, Alberta, Canada, September 2-7, 1990. 
The hydrogen is trapped at its site by its own distortion field (self-trapping). Periodicity is of course conserved for the hydrogen dressed with its distortion field, the so-called small polaron.

The vibrational spectrum of hydrogen in transition metals is split into two parts. Because of its small mass hydrogen mainly vibrates with localized modes, far above the band of lattice modes. In the case of $\mathrm{Nb}-\mathrm{H}$, for example, $99 \%$ of the vibrational spectrum is taken by these localized (optical) modes. Nevertheless, the temperature dependence of the hydrogen dynamics below room temperature is governed by the modes in the band of the host frequencies (band modes). Their strength depends strongly on the local geometry. In the case of the $\mathrm{Nb}-\mathrm{H}$ the hydrogen amplitudes are strongly enhanced for some modes (reasonant-like behaviour) $[2,3]$. At $T=400 \mathrm{~K}$ the mean square displacements of hydrogen due the band modes exceed those caused by the localized modes. These "resonant" modes couple mainly to the short wavelength phonons. In the $\beta$ phase they merge to low lying optic modes [4].

As regards the motion of the hydrogen between interstitial sites, various temperature regimes have to be distinguished. In the limit $T \rightarrow 0 \mathrm{~K}$ all phonon and electron degrees of freedom are frozen and the hydrogen can only move by coherent tunnelling. In this process the hydrogen plus its surrounding displacement field and its electron cloud move from one site to an equivalent site without excitation of phonons or electron-hole pairs. With increasing temperature more and more phonon and electron states are excited and coherent tunnelling between such states will occur; the tunnelling rate becomes temperature dependent. However, already at very low temperatures transitions between non-equivalent configurations, i.e. with excitations of phonons and electron-hole pairs, will dominate owing to the greater number of available transition channels. Since the vibrational density of states vanishes as $Z(v) \propto v^{2}$ for small $v$ whereas the electron density of states is finite at the Fermi level, the electronic excitations dominate initially before the phonons take over [5]. The strength of the electronic contribution can be deduced from the change in the tunnel splitting at the transition to superconductivity [6]. The first phonons to be excited are the long-wavelength phonons. In this regime the transition rates can be calculated by path integral techniques assuming that these phonons are coupled linearly to the hydrogen position $[7,8]$. Assuming Debye phonons and an effective tunnelling element, analytic results can be obtained. If the temperature is increased further, the detailed structure of the coupled hydrogen metal vibrational system becomes important until finally the localized vibrations are excited.

We have developed a method to calculate transition rates in this temperature regime [9]. In the following we shall give a short outline of our method and compare it with related work. In a final section results for $\mathrm{Nb}-\mathrm{H}$ and $\mathrm{Lu}-\mathrm{H}$ are presented, one a typical b.c.c. system and the other h.c.p.

\section{Calculation of transition rates}

The calculation of transition rates is based on the idea that one can 
define sensibly localized states between which the transitions occur. We assume that the hydrogen is localized at one site for a sufficiently long time for self-trapping to be effective. The transition probability can then be found by use of the Fermi golden rule expression for quantal transition rates:

$$
\begin{aligned}
W^{\mathrm{if}} & =\left\langle\left\langle w^{\mathrm{if}}\right\rangle\right\rangle_{m} \\
w^{\mathrm{if}} & =(2 \pi / \hbar)\left|\left\langle\Psi_{\mathrm{i}}(m)\left|H-E_{\mathrm{i} m}\right| \Psi_{\mathrm{f}}(n)\right\rangle\right|^{2} \delta\left(E_{\mathrm{i} m}-E_{\mathrm{f} n}\right)
\end{aligned}
$$

Here $\Psi_{\mathrm{i}}$ and $\Psi_{\mathrm{f}}$ are the initial and final state many-body wavefunctions and $m$ and $n$ denote vibrational states, with $E_{\mathrm{i} m} \equiv\left\langle\Psi_{\mathrm{i}}(m)|H| \Psi_{\mathrm{i}}(m)\right\rangle$. The thermal average over initial states $m$ and summation over final states $n$ is written $\langle\langle\rangle\rangle_{m}$.

The validity of this expression is restricted to the purely incoherent regime. Different methods have to be employed to study the transition from coherent to incoherent tunnelling [7]. Also correlations between single jumps which become important at high temperatures are not included. The various terms in eqn. (2) involve all phonon and all electron degrees of freedom and clearly cannot be calculated without some approximations. First the standard Born-Oppenheimer approximation of phonon physics is used. One assumes the electrons to follow the ions instantaneously and their effect to be included in the ion-ion interaction. The residual electron dynamics, which gives rise to the anomalies at very low temperatures, is factored out $[5,8]$. At temperatures $T \geqslant 40 \mathrm{~K}$ the effect of the electronic degrees of freedom can be dealt with by a slight broadening of the $\delta$-function in eqn. (2). Since we are interested in the phonon effects we will neglect the electron dynamics. The standard procedure by Flynn and Stoneham [10] to solve the phonon part of eqn. (2) invokes three main working assumptions: (i) an adiabatic approximation to decouple the hydrogen and lattice degrees of freedom; (ii) the assumption of linear coupling between phonons and defect position, which means that the phonons are merely hydrogen displaced in the transition but not altered; (iii) the Condon approximation that the hydrogen tunnelling element is independent of the phonon occupation. This results in the simple expression

$$
W^{\text {if }}=(2 \pi / \hbar)|J|^{2}\left\langle\left\langle\left|\sigma^{\text {if }}\right|^{2}\right\rangle\right\rangle_{m}
$$

Here $J$ is the tunnel splitting of the hydrogen in the fixed potential of the host ions and $\sigma^{\text {if }}$ is the overlap of the pure host ion wavefunctions. In the high temperature limit, this takes the form

$$
W^{\text {if }}=\left(\pi / 4 \hbar^{2} E_{\mathrm{c}} k T\right)^{1 / 2}|J|^{2} \exp \left(-E_{\mathrm{c}} / k T\right)
$$

$E_{\mathrm{c}}$ is the so-called coincidence energy, i.e. the minimal energy necessary to deform the lattice such that the initial and final configurations (hydrogen plus host) are equivalent. At intermediate temperatures the Arrhenius-like behaviour of $W$ is modified by a temperature-dependent factor to $E_{\mathrm{c}}$ [11].

The above approximations will break down at temperatures where the localized hydrogen states become markedly excited. This could be taken into account by introducing a thermal average for $J$. Considering the strong 
coupling between the hydrogen and the host phonons we expect deviations from the above approximations already at much lower temperatures. This breakdown of the Condon and adiabatic approximations can be studied more easily using an expression for the coherent tunnelling transitions which involves fewer summations. The thermal average of the tunnel frequency can be expressed by a formula similar to eqn. (2):

$$
\left\langle h v_{\mathrm{T}}\right\rangle_{m} \approx(2 \pi / h)\left\langle\Psi_{\mathrm{i}}(m)\left|H-E_{\mathrm{i} m}\right| \Psi_{\mathrm{f}}(m)\right\rangle_{m}
$$

where $\mathrm{i}$ and $\mathrm{f}$ denote equivalent states. Employing the simplifications leading to eqn. (4) in the case of incoherent transitions simplifies eqn. (5) to

$$
\left\langle h v_{\mathrm{T}}\right\rangle_{m}=J\left\langle\sigma^{\mathrm{if}} \mid\right\rangle_{m}=J \exp (-S / 2)
$$

Here $S$ is the Huang-Rhys factor known from the theory of radiationless transitions [12]. The total dressing $\exp (-S / 2)$ of the tunnelling element is of order 0.1 for $\mathrm{Nb}-\mathrm{H}$. For isotropic defects one has $S(T)=S(0)+\alpha T^{4}$.

Using methods described elsewhere [13] we have evaluated eqn. (5) and the simplified expression (6) for $\mathrm{Nb}-\mathrm{H}$ using the interaction model of Sugimoto and Fukai [14]. In Fig. 1 we show the temperature dependence of $v_{\mathrm{T}}$ for the two expressions. For low temperatures we find agreement between the two expressions but for $T>40 \mathrm{~K}$ the full expression (5) predicts a strong isotope-dependent structure absent in the simplified form of eqn. (6). This indicates a breakdown of the adiabatic and Condon approximations at temperatures where the resonant-like (in-band) vibrations of the hydrogen are excited. The same effects are apparent in the variance of $v_{\mathrm{T}}$ which increases rapidly with temperature. At $T \approx 50 \mathrm{~K}$ it reaches $50 \%$. In passing we want to note that an estimate of $S$ from isotropic elasticity theory seriously underestimates $S$ by about a factor of 5 which again shows that the bulk of the dressing stems from short-wavelength phonons.

There have been numerous attempts to avoid the serious approximations, in particular the Condon approximation. In the "occurrence probability" method $[15,16]$ the adiabatic approximation is retained but one averages over possible coincidence configurations. Also transitions between different excitation levels of the localized hydrogen states are included. Since different coincidence configurations have different tunnelling probabilities for the "naked" hydrogen, an additional temperature dependence is found which can explain the change in slope of the Arrhenius plot for diffusion of hydrogen in niobium. Gillan [17] uses quantum molecular dynamics to identify the symmetric coincidence configurations. A direct calculation of the transition rate is not yet possible and the influence of asymmetric coincidence configurations is difficult to estimate. Both methods allow a comparison with classical diffusion.

We use a different approach. We make use of the spatial localization of the resonant and localized modes. We do not separate the hydrogen and host degrees of freedom but treat a cluster of atoms (typically 6-21 atoms) explicitly and use the above approximations only for the embedding of the cluster into the rest of the host crystal [9]. The limitations of our method are mainly due to computer capacity. The number of degrees of freedom we can 


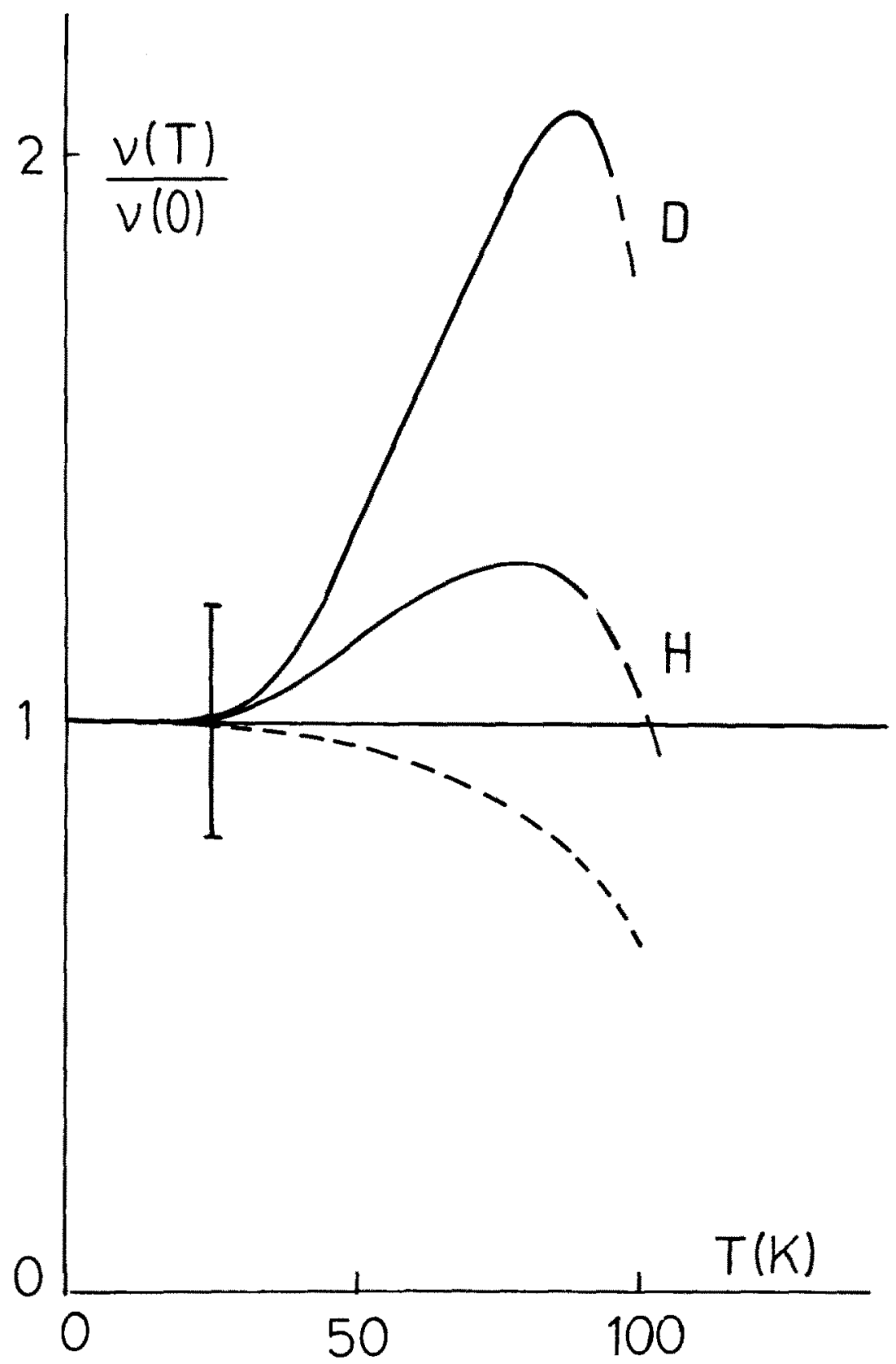

Fig. 1. Temperature dependence of tunnelling rate for hydrogen and deuterium in niobium calculated from eq. (5) (_- - ) and in the weak coupling approximation of eqn. (6) (- -). 
treat explicitly is limited owing to the number of integrations involved in calculating a single transition element $w^{\text {if }}$ in eqn. (2). On the temperature side we are limited by the rapid increase in terms $w^{\text {if }}$ contributing to eqn. (1). Our accuracy is further limited by the strong dependence on the chosen basis functions. In order to avoid numerical integration errors we take harmonic vibrational wavefunctions as the basis. For these the single transition terms can be evaluated exactly. This basis is sufficient to give a qualitative picture of quantum diffusion. In order to obtain a quantitative description the anharmonicity of the local modes and the anharmonic coupling between modes have to be included. These corrections become more important with increasing temperature and increasing isotopic mass.

\section{Results}

In Fig. 2 we compare our results for the $\mathrm{Nb}-\mathrm{H}$ system [9] in the harmonic approximation and with anharmonic corrections to the experimental data [18]. The importance of the anharmonicity for the magnitude of the diffusion constant is evident. Our low temperature activation energy is much lower then the experimental value. However, in recent nuclear magnetic resonance experiments [19] a value of $37 \mathrm{meV}$ was found which would be in much better agreement with our value of about $30 \mathrm{meV}$. As in experiment we find a change in slope at about $T=250 \mathrm{~K}$ for $\mathrm{Nb}-\mathrm{H}$. For $\mathrm{Nb}-\mathrm{D}$ we predict a similar change at about $T=150 \mathrm{~K}$. The origin of these kinks can be understood from the partial rates $w^{\text {if }}$. At low temperatures localized mode excitations can be ignored: the lowest coincidence energy dominates and the observed activation energy is close to the coincidence energy. At higher temperatures the lattice modes have enough energy to scatter into the localized modes and new and effective transition channels (ground state to excited state) are opened. Eventually the excited states of the localized modes become populated. Because of the lower frequencies of these modes for the heavier isotopes the cross-over between the two regimes is shifted to lower temperatures and is less acute. Since the tunnelling rates are lower the gain for higher excited states is more effective and the high temperature activation is slightly increased. The same interplay between lattice activation and localized mode excitation should lead to an anomaly in the pressure dependence of the diffusion constant [20].

Our interpretation is in agreement with the results of the occurrence approach [15, 16]. A difference from the work of Vargas et al. [21] is that we do not introduce excited states as new parameters. The analysis of the anharmonicity shows that, at least for the interaction model used, the excited localized states are well defined and well localized to the tetrahedral site. $\mathrm{Nb}-\mathrm{H}$ is a system where the classical saddle point has an energy lower than or similar to the excitation energy of the localized modes for the tetrahedral configuration. In such a situation lattice deformations can lead to configurations where the excited state hydrogen wavefunction is no longer localized to 


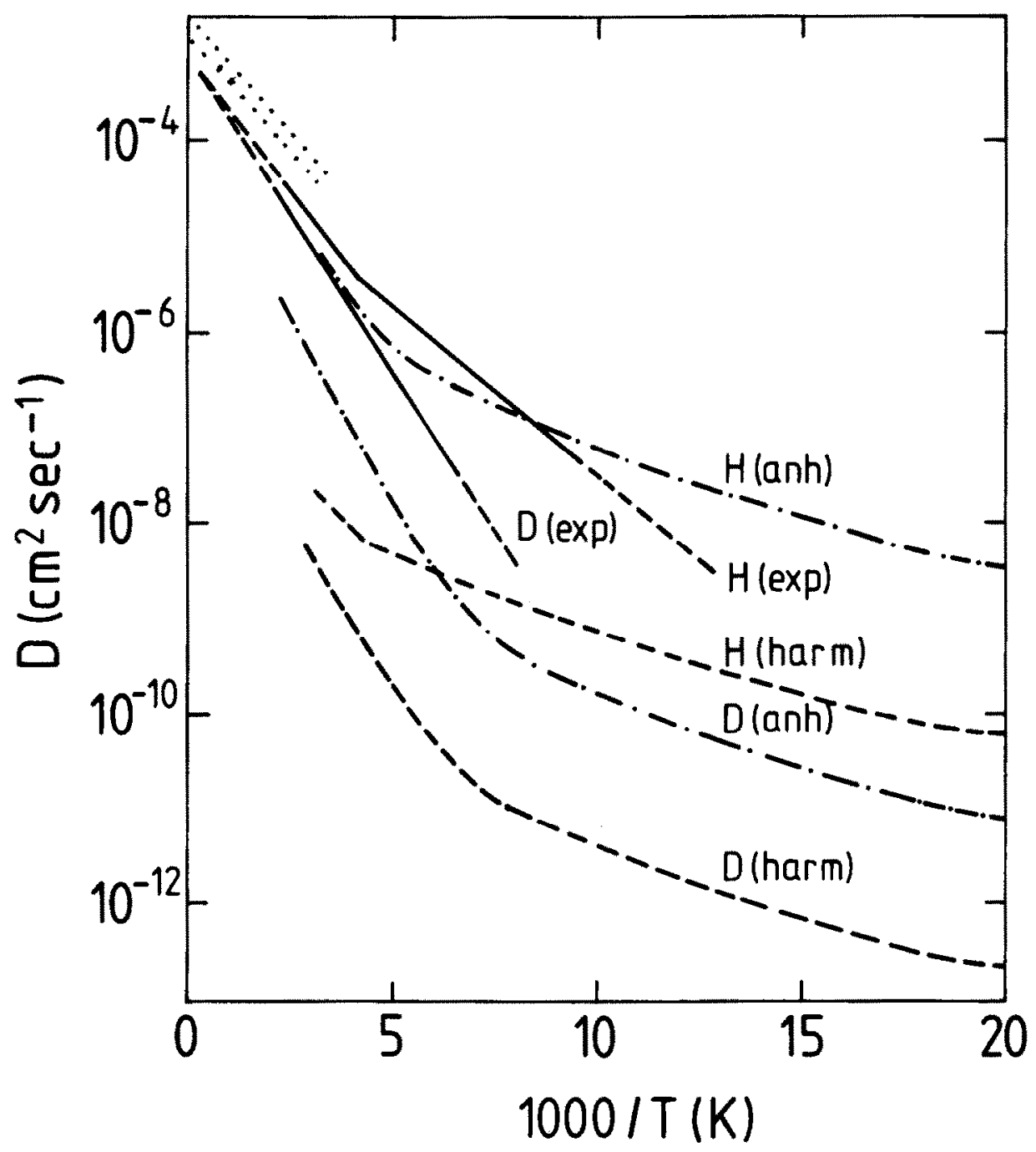

Fig. 2. Diffusion constants of hydrogen and deuterium in niobium: -- , experimental results [18]; - - - calculation with harmonic wavefunctions; $-\cdot-$, calculation including anharmonic corrections; ..... , Vineyard approximation.

the tetrahedral site and where the total energy of this configuration is lower than the corresponding energy for the tetrahedral site. This becomes plausible if one considers that one of the localized vibration modes has to become unstable at the saddle point.

According to our model the diffusion of hydrogen at room temperature is still influenced strongly by quantum mechanics. Our calculation is not sufficiently accurate to see whether and how the classical limit is reached at higher temperatures. For comparison we show in Fig. 2 also the results of a 
calculation of the Vineyard approximation [22] for classical diffusion using the same interaction model as in the quantum calculation. The shift in the prefactor between hydrogen and deuterium is about what one would expect from the mass difference. The activation energy is $97 \mathrm{meV}$ and $96 \mathrm{meV}$ for hydrogen and deuterium respectively. Here $90 \mathrm{meV}$ is the potential energy part (the value of $61 \mathrm{meV}$ quoted earlier refers to a different cut-off radius in the $\mathrm{Nb}-\mathrm{H}$ potential). The discrepancy between our quantum results and the classical results is not sufficient for us to draw any definite conclusion. Gillan [17] puts the classical-to-quantum transition already at the kink. Since there might be a smooth transition and because of differences in the model this need not be in contradiction to our results.

Contrary to $\mathrm{Nb}-\mathrm{H}$, where over a large temperature range quantum effects are dominant, $\mathrm{Pd}-\mathrm{H}$ behaves more like a classical diffusion system [1]. This is due to the large distance between the octahedral equilibrium configurations, which suppresses the tunnelling exponentially. Additionally the localized modes are at much lower frequency and the classical limit should, therefore, be reached at lower temperatures. Quantum effects are expected only for the light $\mu^{+}$. The diffusion of hydrogen and deuterium is thought to be classical, with minor corrections, via metastable tetrahedral configurations. In a rough calculation we did not find any sizeable contribution from direct tunnelling between octahedral sites.

In h.c.p. transition metals the situation is more complicated. The hydrogen is thought to occupy tetrahedral interstitial sites [23]. The diffusion involves a jump between nearest-neighbour tetrahedral sites and another jump going to octahedral sites [24]. At least for the first of these jumps the geometry is similar to that familiar from the b.c.c. metals. To investigate the quantum effect for this jump we studied a simple model of $\mathrm{Lu}-\mathrm{H}$. The interaction model was similar to that employed for $\mathrm{Nb}-\mathrm{H}$, namely we described the $\mathrm{Lu}-\mathrm{Lu}$ interaction by the measured Born-von Karman parameters [25] and used for the $\mathrm{H}$ - $\mathrm{Lu}$ interaction the potential of Sugimoto and Fukai for $\mathrm{H}-\mathrm{Nb}$ [14] with the $\mathrm{H}-\mathrm{Lu}$ instead of the $\mathrm{H}-\mathrm{Nb}$ nearestneighbour distance. This non-optimized model already gives quite good results for the localized modes: $h v=75$ and $96 \mathrm{meV}$ for deuterium compared with the experimental values [26] of $h v=70$ and $100 \mathrm{meV}$. The large split between the mode along the hexagonal axis and those in the basal plane is caused by lattice relaxation. Figure 3 shows our results for the jump rates between the nearest-neighbour tetrahedral sites. The calculation was carried out in the harmonic approximation. As in $\mathrm{Nb}-\mathrm{H}$, a change from low to high temperature behaviour is found, with a crossover at about $T=125 \mathrm{~K}$. Above that temperature the rate increases rapidly. The large rate at $T=500 \mathrm{~K}$ is in agreement with experiments on lutetium [24] and on yttrium [27]. Our present calculation is not intended to give exact values but only to show the qualitative behaviour. Judging from $\mathrm{Nb}-\mathrm{H}$ our values should seriously underestimate the jump rate. We also did not include effects of the zero-point vibrational energy on the lattice relaxation. 


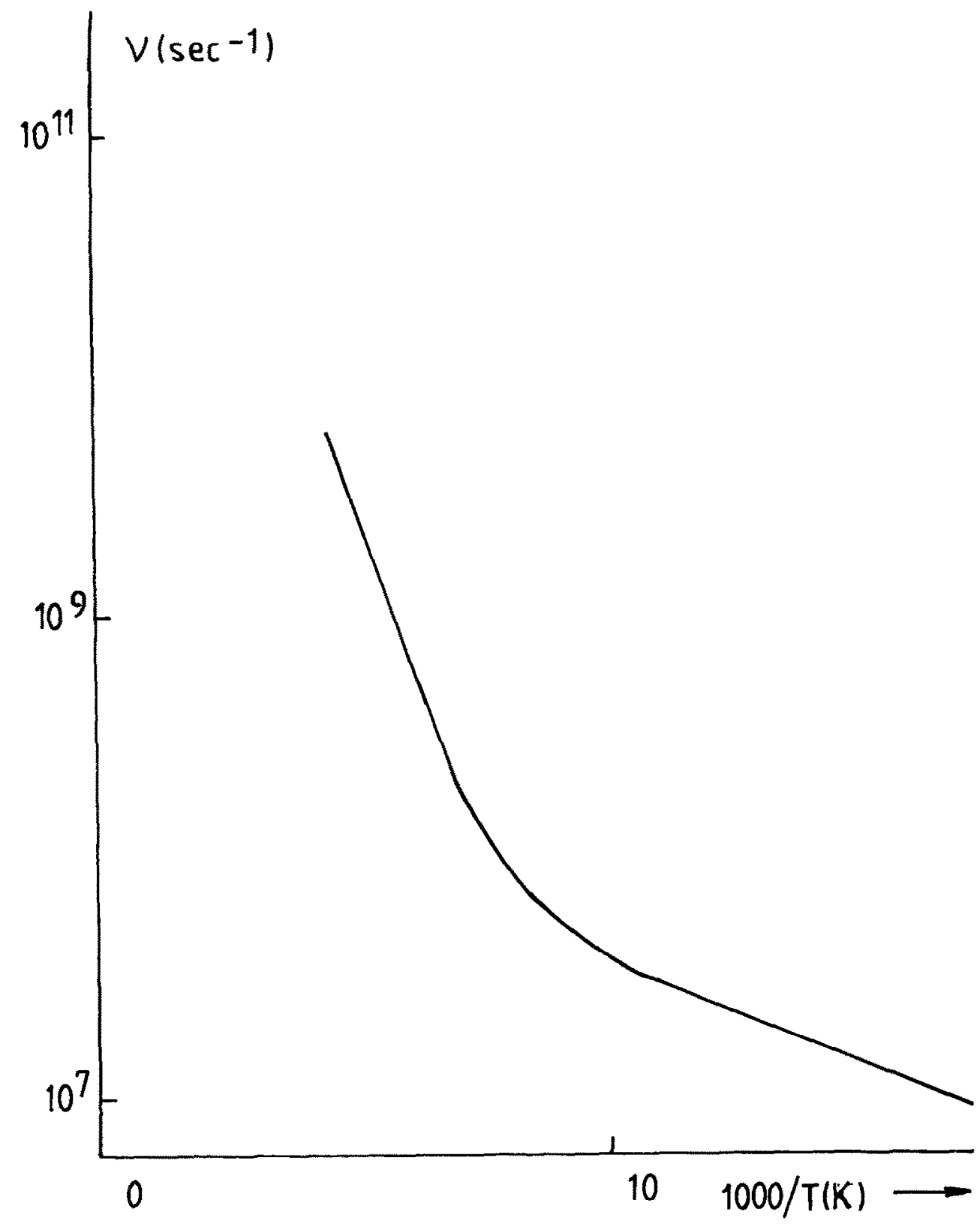

Fig. 3. Jump rate between nearest-neighbour tetrahedral sites for hydrogen in lutetium in the harmonic approximation. 


\section{Conclusion}

We have presented a model which is a systematic generalization of the Flynn-Stoneham model. It successfully describes diffusion of hydrogen in niobium. The model is devised to take the strong dynamic interaction between defect and host into account. At low temperature it merges into the usual weak coupling models which allow the low temperature effects to be studied. Our model is applicable to situations where a few modes determine the defect dynamics. We have shown that quantum effects are important for the diffusion of hydrogen in b.c.c. and h.c.p. transition metals.

\section{References}

1 Y. Fukai and H. Sugimoto, Adv. Phys., 34 (1985) 263.

2 V. Lottner, H. R. Schober and W. J. Fitzgerald, Phys. Rev. Lett., 42 (1979) 1162.

3 H. R. Schober and V. Lottner, Z. Phys. Chem., N.F., 114 (1979) 203.

4 A. Magerl, J. M. Rowe and D. Richter, Phys. Rev. B, 23 (1981) 1605.

5 J. Kondo, Physica B, 125 (1984) 279.

6 A. Magerl, A. J. Dianoux, H. Wipf, K. Neumaier and I. S. Andersen, Phys, Rev. Lett., 56 (1986) 159 .

H. Wipf, D. Steinbinder, K. Neumaier, P. Gutsmiedl, A. Magerl and A. J. Dianoux, Europhys. Lett., 4 (1987) 1379.

7 A. O. Caldeira and A. J. Leggett, Ann. Phys. (NY), 149 (1983) 374.

H. Grabert, U. Weiss and P. Hanggi, Phys. Rev. Lett., 52 (1981) 2193.

A. J. Legget, S. Chakravarty, A. T. Dorsey, M. P. A. Fisher, A. Garg and W. Zwerger, Rev. Mod. Phys., 59 (1987) 2.

8 H. Grabert, U. Weiss and H. R. Schober, Hyperfine Interact., 31 (1986) 147.

9 H. R. Schober and A. M. Stoneham, Phys. Rev. Lett., 60 (1988) 2310.

H. R. Schober and A. M. Stoneham, Z. Phys. Chem., N.F., 164 (1989) 757.

10 C. P. Flynn and A. M. Stoneham, Phys. Rev. B, 1 (1970) 3966.

A. M. Stoneham, Ber. Bunsenges. Phys. Chem., 76 (1972) 816.

11 M. Lagos, Solid State Commun., 50 (1984) 777.

12 A. M. Stoneham, Theory of Defects in Solids, Clarendon, Oxford, 1975.

13 H. R. Schober and A. M. Stoneham, Phys. Rev. B, 26 (1982) 1819.

14 H. Sugimoto and Y. Fukai, Phys. Rev. B, 22 (1980) 670.

15 D. Emin, M. J. Baskes and W. D. Wilson, Phys. Rev. Lett., 42 (1979) 791.

16 A. Klamt and H. Teichler, Phys. Status Solidi B, 134 (1986) 103, 533.

17 M. J. Gillan, Phys. Rev. Lett., 58 (1987) 563.

18 Z. Qi, J. Völkl, R. Lässer and H. Wenzl, J. Phys. F, 13 (1983) 2053.

19 R. Messer, A. Blessing, S. Dais, D. Höpfel, G. Majer, C. Schmidt, A. Seeger, W. Zag and R. Lässer, Z. Phys. Chem., N.F., Suppl. H2 (1984) 62.

20 D. Steinbinder, H. Wipf, H. R. Schober, H. Blank, G. Kearley, C. Vettier and A. Magerl, Europhys. Lett., 8 (1989) 269.

21 P. Vargas, L. Miranda and M. Lagos, Z. Phys. Chem., N.F., 164 (1989) 975.

22 G. H. Vineyard, J. Phys. Chem. Solids, 3 (1957) 121.

23 H. D. Carstanjen, Z. Phys. Chem., N.F., 164 (1989) 141.

24 J. Völkl, H. Wipf, B. J. Beaudry and K. A. Gschneidner, Phys. Status Solidi B, 144 (1987) 315.

25 J. Pleschiutschnig, O. Blaschko and W. Reichardt, Phys. Rev. B, 41 (1990) 975.

26 O. Blaschko, G. Krexner, L. Pintschovius, P. Vajda and J. N. Daou, Phys. Rev. B, 38 (1988) 9612.

27 I. S. Anderson, D. K. Ross and J. E. Bonnet, Z. Phys. Chem., N.F., 164 (1989) 923. 